\title{
Metodologías activas en asignaturas básicas. Creación de un catálogo de demostraciones experimentales o proyectos como recursos didácticos para la motivación de título (Fase II)
}

\author{
Rosario Vilaplana Cerdáa, Romina Del Rey Tormosa, Francisco Javier Manjón \\ Herrera $^{\mathrm{b}}$, Juan Antonio Monsoriu Serra ${ }^{\mathrm{b}}$, Pedro LLovera Segovia ${ }^{\mathrm{c}}$ y Constantino \\ Torregrosa Cabanilles ${ }^{\mathrm{d}}$
}

\begin{abstract}
âniversitat Politècnica de Valéncia, Escuela Politécnica Superior de Alcoy, Departamento de Física Aplicada, Pz. Ferràndiz i Carbonell s/n - 03801 Alcoy (Alicante). rovilap@fis.upv.es, roderey@doctor.upv.es
\end{abstract}

bUniversitat Politècnica de Valéncia, Escuela Politécnica Superior de Diseño, Departamento de Física Aplicada, Camino de Vera s/n - 46022 Valencia. fjmanjon@fis.upv.es, jmonsori@fis.upv.es

\footnotetext{
'Universitat Politècnica de Valéncia, Escuela Técnica Superior de Caminos, Canales y Puertos, Departamento de Ingeniería Eléctrica, Camino de Vera s/n - 46022 Valencia pllovera@ite.upv.es
}

${ }^{\mathrm{d}}$ Universitat Politècnica de Valéncia, Escuela Politécnica Superior de Gandia, Departamento de Física Aplicada, C/Paraninfo n¹ - 46715 Grao de Gandia. ctorregr@fis.upv.es

\begin{abstract}
We present the steps carried out within a PIME entitled "Active methodologies in basic subjects. A catalogue of experimental Physics demonstrations designed as teaching resources for the degree motivation" that started in 2016 and will continue until 2019. This project aims at designing a catalogue of simple experimental Physics demonstrations made with readily available low cost materials. These resources are intended to be used in theory classes and problems to demonstrate physical phenomena and their relationship to the theoretical models, thus favouring the understanding of phenomena. During this second phase of the project, the catalog of experimental demonstrations designed by the teachers has continued to be expanded. But in addition, two activities have been carried out in which is prioritized the direct experimentation with the concepts and the most practical elements by the students, so that the students take greater role being promoted in this way the studentcentered learning, the active methodologies and the small project-based learning.
\end{abstract}


Keywords: Active Methodologies, Motivation, Competencies, ProjectBased Learning.

\begin{abstract}
Resumen
Presentamos los pasos realizados dentro de una PIME denominado "Metodologías activas en asignaturas básicas. Un catálogo de demostraciones experimentales de física diseñado como recursos didácticos para la motivación del grado" que arranca en el 2016 y continuará hasta 2019. En este proyecto se está elaborando un catálogo de demostraciones experimentales sencillas hechas con materiales fácilmente disponibles y de bajo coste. Estos recursos están destinados a ser utilizados en las clases de teoría y problemas para demostrar los fenómenos físicos y su relación con los modelos teóricos, favoreciendo así la comprensión de los mismos. Durante la segunda fase del proyecto se ha continuado ampliando el catálogo de demostraciones experimentales diseñadas por los profesores. Pero además se han realizado dos actividades en las que la experimentación directa con los conceptos y los elementos más prácticos por parte del alumnado se prioriza, de modo que el alumno toma más protagonismo, promoviéndose de este modo el aprendizaje más centrado en el estudiante, las metodologías activas y el aprendizaje basado en pequeños proyectos.
\end{abstract}

Palabras clave: Metodologías Activas, Motivación, Competencias, Aprendizaje Basado en Proyectos.

\title{
Introducción
}

En este trabajo se exponen los avances en la segunda fase del desarrollo del Proyecto de Innovación y Mejora Educativa (PIME) titulado: "Metodologías activas en asignaturas básicas. Creación de un catálogo de demostraciones experimentales o proyectos como recursos didácticos para la motivación de título” y cuya primera fase ya fue desarrollada durante el curso 2016-2017 (Vilaplana 2017). Este proyecto está enfocado a la creación e implementación de un catálogo de demostraciones experimentales de Física y a promover el uso de metodologías activas en las que se mezcle la teoría con sesiones más prácticas en el aula.

La presentación de los conceptos teóricos y la deducción de las teorías son siempre positivas y necesarias, pero la teoría se entiende mejor si se conecta inmediatamente con una experiencia práctica (Freier 1996). Demostraciones prácticas del profesor en el aula pueden ayudar en esta tarea; de hecho, este proyecto tiene como uno de sus objetivos prioritarios el desarrollo cooperativo entre profesores para implementar demostraciones experimentales de física diseñadas como recursos didácticos para la motivación del grado (Vilaplana 2017). Las demostraciones experimentales de Física u otras disciplinas forman parte de la práctica docente de diferentes países y su uso está recogido

(cc) BY-Nc-No 2018, Universitat Politècnica de València

Congreso IN-RED (2018) 
explícitamente en el proyecto europeo "Tunning" de Física para la armonización de titulaciones universitarias (González 2003). No obstante, actualmente su uso en nuestro país, no forma parte de las prácticas docentes generalizadas, salvo excepciones (Ferrer 2012). En este proyecto se realizan acciones encaminadas a corregir esta situación.

Las lecciones teóricas y las sesiones de laboratorio están, por lo general, separadas por razones prácticas. Las sesiones de laboratorio son más caras que las lecciones en el aula y el tamaño del grupo para el trabajo de laboratorio tiene que ser pequeño. Como resultado, existe una separación artificial entre la teoría y la práctica que no es adecuada especialmente para la ingeniería. En general, la ingeniería es una disciplina práctica pero con un amplio contenido de contenidos teóricos (Freisel 2005). Así, el equilibrio entre la teoría y la práctica no es fácil de definir, y es una sensación muy común que la teoría ha ganado peso relativo respecto al laboratorio. Sin embargo, esta separación es artificial; y por tanto, debería haber una cooperación entre ambos campos.

Por otro lado, es sabido que la experimentación directa del estudiante con los conceptos y elementos prácticos conduce a mejores resultados en la comprensión y el aprendizaje. En muchos campos de la ingeniería, la simulación por ordenador de la situación práctica ha tomado un lugar preponderante en las sesiones prácticas. Estas herramientas de software son de primordial importancia para los estudiantes, ya que tendrán que utilizarlas en el futuro inmediato; no obstante, el contacto directo con los experimentos físicos no puede ser sustituido por instrumentos de simulación, especialmente durante los primeros cursos. Esto nos lleva al segundo objetivo del proyecto que consiste crear actividades o pequeños proyectos que permitan la experimentación directa del estudiante durante las sesiones en el aula. Para ello hacemos uso del aprendizaje basado en pequeños proyectos (Calvo 2010, Alba 2015) y llegamos a la explicación de los conceptos de una manera más natural, transformando de este modo la típica clase magistral en una clase dialogada y preguntada (Cury 2007). A este respecto, ya hemos realizado dos actividades con los alumnos en clase, en la que se prioriza la experimentación directa con los conceptos y los elementos más prácticos.

La primera de estas actividades se ha llevado a cabo con los alumnos de primero en el Grado en Ingeniería en Diseño Industrial y Desarrollo de Productos. Estos alumnos han diseñado algunas demostraciones experimentales o pequeños proyectos en grupo con la guía del profesor. Estas demostraciones experimentales se caracterizan por el uso de materiales fácilmente asequibles y de bajo coste que pueden ser diseñados sin coste, usando materiales reciclados y fácil de obtene. Con ello, se quiere fomentar la capacidad de sorpresa y articular un proceso de observación racional, algo esencial para la comprensión del método científico, en el que los alumnos emitan conclusiones en términos de los modelos físicos que se estudian en el ámbito teórico de asignaturas básicas como la Física. También se pretende trasmitir a los alumnos de esta titulación, el hecho de que aquellos diseños que incluye ciencia, cobrán un valor añadido. Ejemplos claros de algunos diseños que existen y que cumplen con estas características son las lámparas de lava o las macetas o lámparas que levitan.

La segunda actividad en esta línea, se ha realizado con los alumnos de segundo año de Ingeniería Civil y de Obras Públicas y de Ingeniería Química en la materia de 
electricidad. La actividad ha consistido en mezclar la teoría y las sesiones de laboratorio en el aula por medio de la implementación de un laboratorio portátil en el aula durante el tiempo asignado a una lección (Llovera 2015). La solución propuesta para la mezcla de teoría y sesiones de laboratorio en el aula, requiere resolver algunas cuestiones: a) el despliegue y la colección del laboratorio necesita un mínimo de tiempo, normalmente, al menos 10 minutos, b) el laboratorio tiene que ser seguro, c) el peso total del equipo que se traslada a las aulas ha de ser tan bajo como sea posible, d) no deben ser equipos que supongan gastos adicionales, sino que contengan equipos disponibles en los laboratorios tales como multímetros, fuentes de alimentación, cables, etc.

\section{Objetivos}

El objetivo original del proyecto es generar recursos útiles y prácticos que ayuden a mejorar y motivar las prácticas docentes en las asignaturas de primeros cursos de Física y Electricidad de distintas Ingenierías. Trascurrido un año y medio del proyecto, los objetivos han ido evolucionando y quedan reflejados del siguiente modo:

1. Seguir desarrollando el catálogo o banco de experimentos disponible para el profesorado que imparte docencia en asignaturas de Física, y continuar de este modo con su uso en las clases de teoría y problemas. Esto conlleva seguir desarrollando los materiales asociados a estas demostraciones tales como fichas, vídeos y cuestionarios.

2. Continuar con la experiencia de trabajar en forma colaborativa los profesores que participan en el proyecto de modo que se generen tres prototipos por cada demostración experimental siempre que sea posible.

3. Involucrar a los alumnos en algunos de estos diseños experimentales en el aula, haciendo así uso de metodologías activas basadas en la realización de proyectos sencillos.

4. Mezclar la teoría y las sesiones de laboratorio de Electricidad en el aula por medio de la implementación de un laboratorio portátil en el aula durante el tiempo asignado a una lección.

5. Hacer especial hincapié en la evaluación de tipo formativo. Lo que hemos comprobado es que los alumnos valoran muy positivamente que se les retroalimente; esto es, que se les de feedback. Así, el desarrollo de competencias se convierte en un fin en sí mismo y dejan a una lado la evalución como una mera herramienta que calificar.

6. Crear rúbricas adecuadas para evaluar los proyectos desarrollados por los alumnos así como los conocimientos y competencias que adquieren.

7. Pasar una encuesta de valoración de metodologías activas a los alumnos con el fin de que muestren su grado de satisfacción y que al mismo tiempo reflejen si ven útil esta práctica docente. De este modo estamos obteniendo información que nos sirve a los profesores no sólo para evaluar el proyecto, sino también para reconducirlo.

(c) B EY-NC-ND 2018, Universitat Politècnica de València

Congreso IN-RED (2018) 


\section{Desarrollo de la innovación}

El desarrollo de la innovación se lleva cabo de modo que:

Cada profesor elabora algunas demostraciones experimentales, desarrollando las correspondientes fichas descriptivas y el posible material asociado, de modo que sirvan de guía a los otros profesores que quieran utilizar dichas demostraciones en clase.

A continuación mostramos en la figura 1 algunas de las demostraciones experimentales desarrolladas por los profesores.

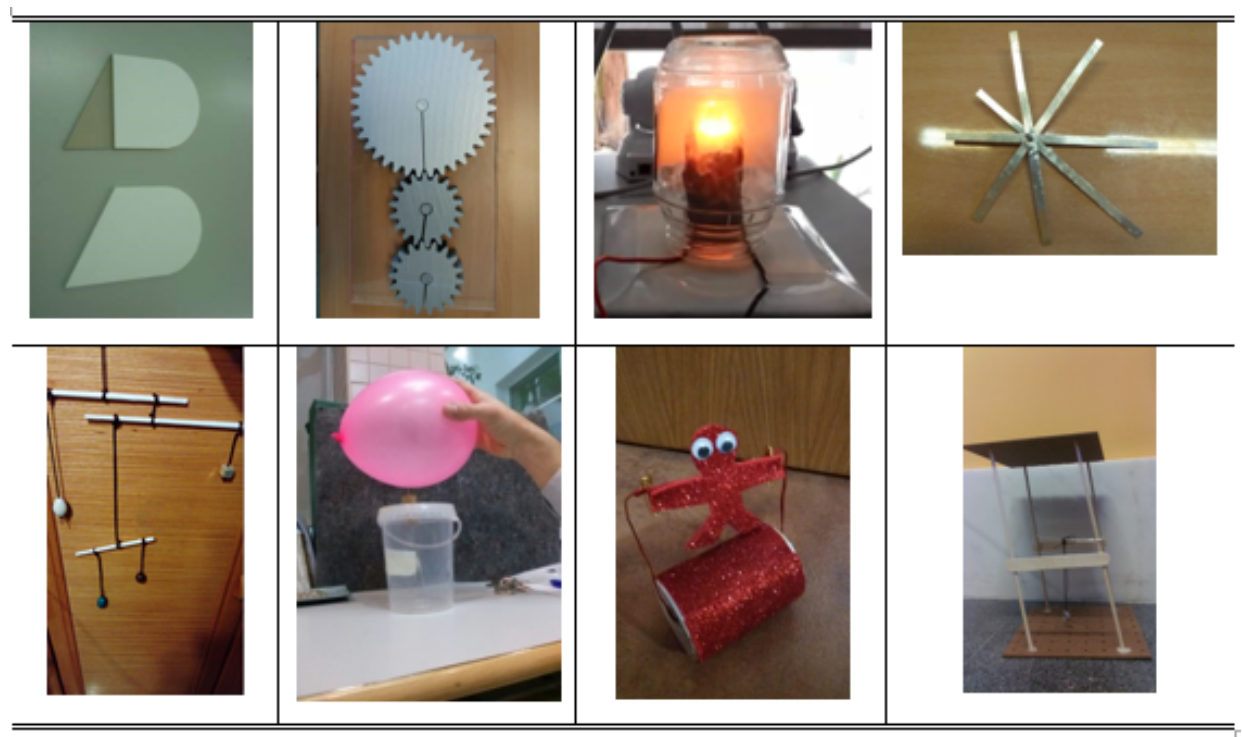

Fig. 1 Ejemplos de demostraciones experimentales desarrolladas por los profesores.

Cada profesor siempre que sea posible genera tres prototipos de cada una de demostraciones experimentales, así los profesores de cada campus puueden disponer de un prototipo.

Seguidamenete describimos como es la ficha estándar a rellenar.

\section{TITULO}

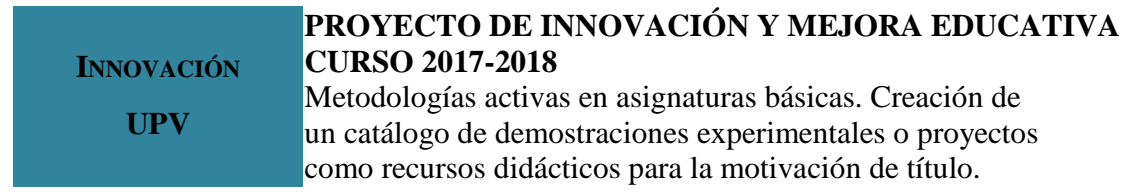

\section{MODALIDAD}

Demostrativa: 


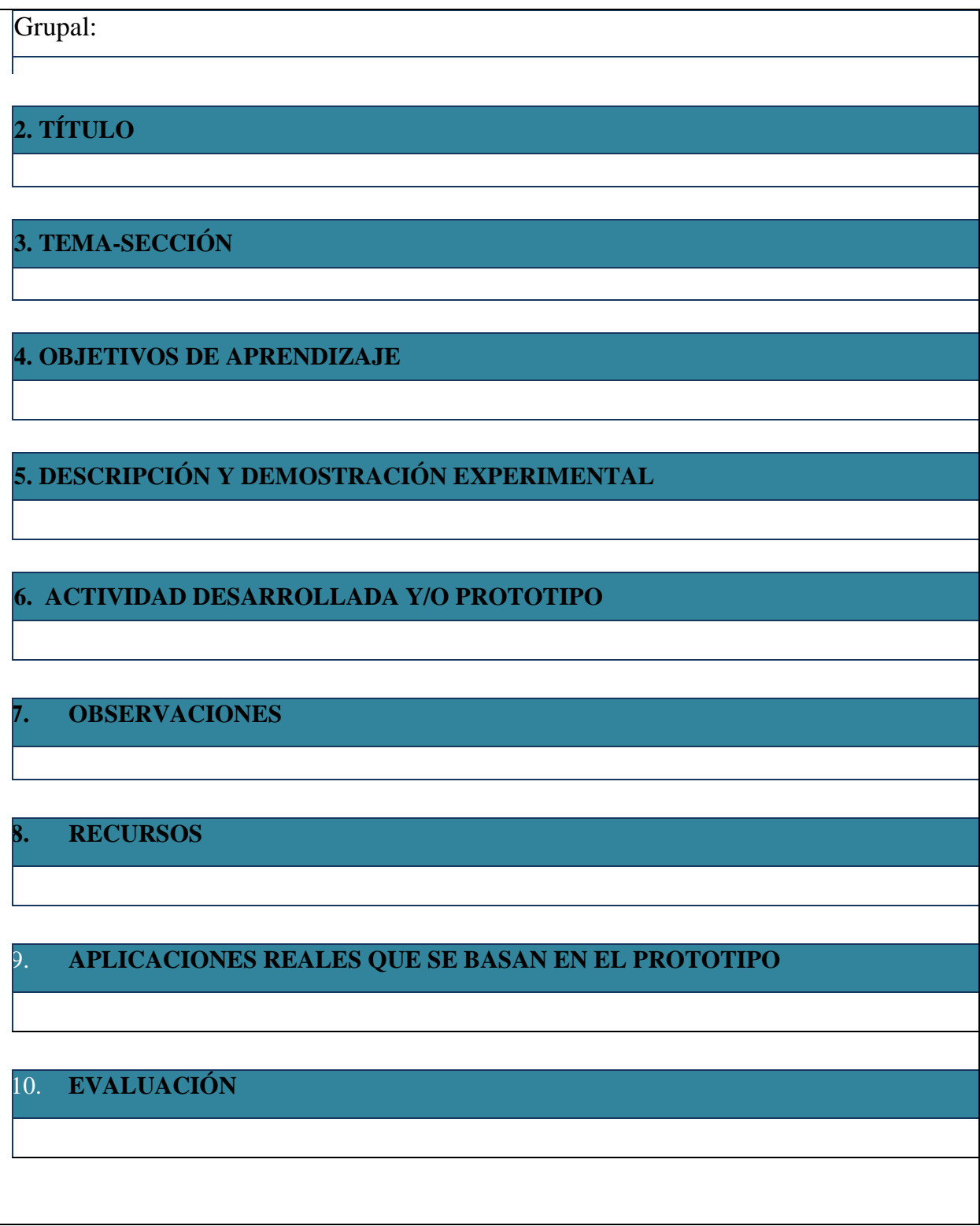

En cuanto a involucrar a los alumnos en alguna de estas demostraciones experimentales, los estudiantes del Grado en Ingeniería en Diseño Industrial y Desarrollo de Productos han desarrollado este año tres pequeños proyectos en grupo con la guía del profesor, a saber: una pieza en 2D para diferenciar el centro geométrico del centro de gravedad, una catapulta y un sistema en equilibrio de varillas con masas colgantes. La figura 2 muestra las imágenes de algunos de prototipos diseñados por los alumnos.

(cc) EY-NC-ND 2018, Universitat Politècnica de València

Congreso IN-RED (2018) 


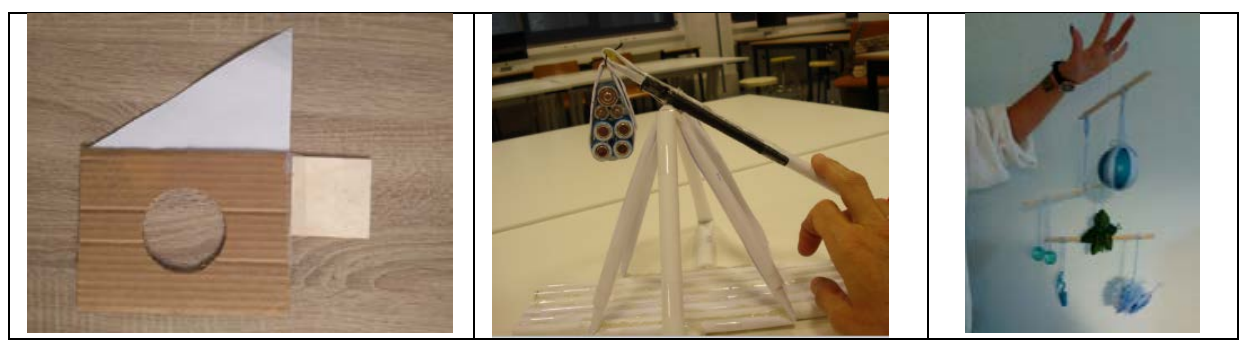

Fig. 2 Ejemplos de los pequeños prototipos desarrollados por los alumnos.

La figura 3 muestra a los alumnos durante una de las sesiones en el aula mostrando los prototipos diseñados por ellos.

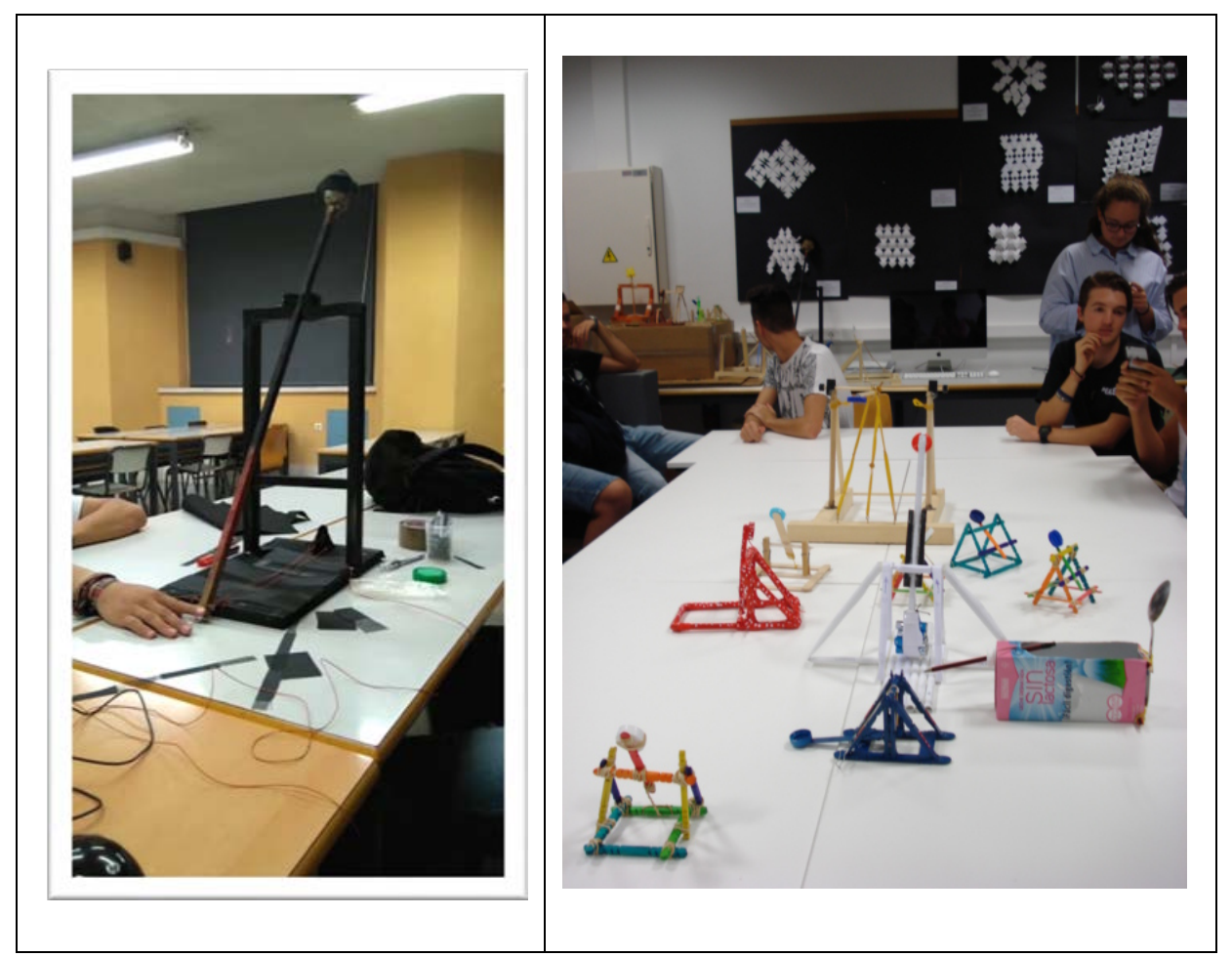

Fig. 3 Los alumnos en el aula durante las demostraciones de sus prototipos.

También se pretende con estos pequeños proyectos que la motivación de los alumnos hacia el aprendizaje de determiandos conceptos físicos sea una consecuencia natural de su experimentación con los prototipos y su diseño. A posteriori, en alguno de estos diseños se les pidio al alumno que resolviese algún problema numérico relacionado con el prototipo y que buscasen ejemplos reales de diseños en donde el prototipo ayudase a la comprensión de su funcionamiento. En la figura 4 se muestra un ejemplo para el caso de las varillas en equilibrio y las masas colgantes. 


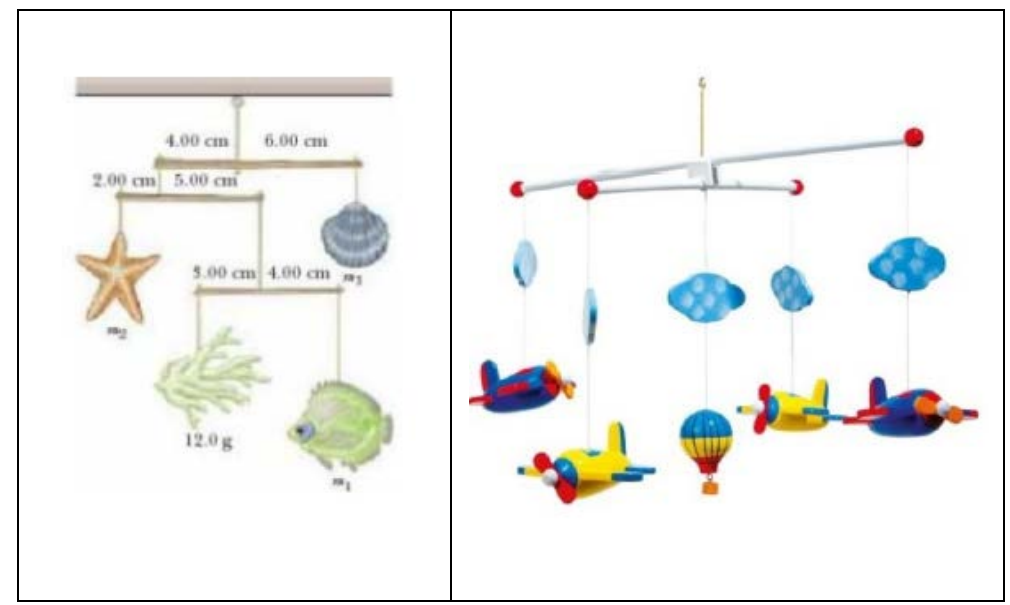

Fig. 4 Problema a resolver y objeto real basado en el equilibrio entre varillas y masas colgantes.

En cuanto al prototipo de la catapulta, además de haber servido para introducir conceptos tales como el tiro parabólico, conservación de energía, momento de inercia de un sólido, teorema de Steiner, etc..., mencionar que fue modelado con el software Matlab (el Matlab es introducido a los alumnos de esta titulación en la asignatura de matemáticas). A partir del modelado hemos observado que ayuda al alumno a identificar los distintos parámetros del mismo y como éstos influyen en su funcionamiento. Entre otros, por ejemplo, les permite calcular la velocidad con la que sale lanzado un proyectil una vez están fijados los otros parámetros modelo (longitud de la barra, peso de la masa lanzada, peso del contrapeso y las longitudes del brazo mayor y menor).

Por último, en relación a la actividad relacionada con la mezcla de la teoría y las sesiones de laboratorio de Electricidad en el aula por medio de la implementación de un laboratorio portátil durante el tiempo asignado a una lección en el aula, se muestra en la figura 6 el kit de trabajo diseñado y a los alumnos trabajando durante una sesión en el aula.

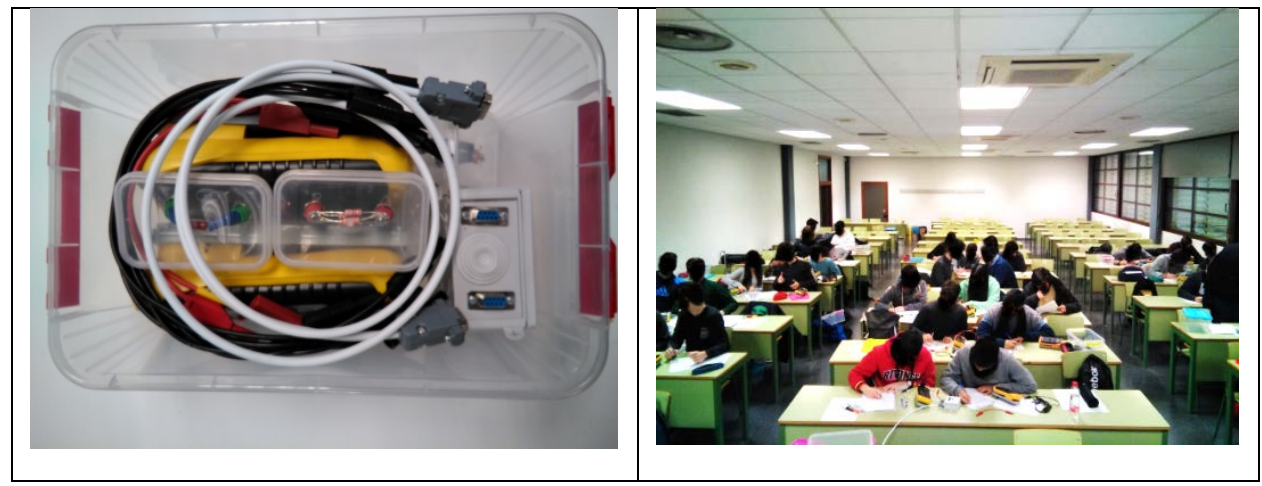

Fig. 6 Kit de cada estudiante y los alumnos trabajando en el aula durante una sesión.

(cc) EY-NC-ND 2018, Universitat Politècnica de València

Congreso IN-RED (2018) 


\section{Resultados}

Algunos de los profesores han pasado una encuesta de valoración para conocer el grado de aceptación por parte de los alumnos de la metodología aplicada.. Se ha considerado interesante utilizar la encuesta que enmarca la valoración a nivel global de toda la Universitat Politécnica de València sobre otro tipo de metodología activa, la docendia inversa (http://docenciainversa.blogs.upv.es/el-proyecto/proyecto-clase-inversa-upv/) . A continuación, mostramos los contenidos de la misma.

\section{Encuesta valoración Metodologías activas en asignaturas básicas: Creación de un catálogo de demostraciones experimentales o proyectos como recursos didácticos para la motivación de titulo}

(Título de la demostración experimental).(Asignatura donde se pasa la encuesta)

Parte de la docencia de esta asignatura ha sido impartida a través de demostraciones experimentales como material de apoyo en las clases de teoría. Se han elaborado unes fichas descriptivas para que los estudiantes pudieseis ver y pensar en fenómenos físicos que explicados desde el punto de vista puramente teórico pudieran quedar "más alejados” de vuestro interés.

Con esto, el profesorado hace uso de las metodologías activas para involucrar a los alumnos en el desarrollo de estas demostraciones experimentales. Se busca que los alumnos tomen iniciativa y protagonismo, desarrollando por grupos, sus propias demostraciones experimentales tutorizadas por el profesor.

\section{EN LAS SIGUIENTE PREGUNTAS VALORA DEL 1 AL 5, TENIENDO EN CUENTA LA} ESCALA:

\section{1: TOTALMENTE EN DESACUERDO... 5: TOTALMENTE DE ACUERDO}

1.- Con la aplicación de estas demostraciones experimentales en clase muestra tu grado de acuerdo con las siguientes afirmaciones:

- Ha contribuido a aumentar mi implicación en la asignatura:

- Me ha resultado gratificante:

- Ha requerido mucho tiempo por mi parte:

- Ha fomentado el trabajo en grupo:

- Me ha ayudado a llegar mejor preparado a los exámenes:

2.- Lo que más te ha gustado de la aplicación de esta metodología en esta asignatura ha sido:

- $\quad$ Los materiales facilitados por el profesor:

- El trabajo realizado en clase:

- El trabajo realizado en casa necesario para llevar a cabo la demostración:

- La relación con el profesor, más cercana y de trabajo conjunto:

- La relación con mis compañeros:

- $\quad$ El sistema de evaluación empleado:

- $\quad$ La preparación de la asignatura por parte del profesor (todo organizado y planificado):

3.- Estoy muy satisfecho con esta experiencia/metodología:

4.- Recomendaría esta experiencia/metodología a mis compañeros de otros cursos y titulaciones:

5.- Me gustaría que esta metodología se aplicara al resto de asignaturas:

6.- Indica dos aspectos positivos y 2 negativos que destacarías de esta metodología:

Positivos:

(cc) EY-NG-ND 2018, Universitat Politècnica de València

Congreso In-Red (2018) 
Negativos:
7.- Añade alguna observación si lo deseas:

En la figura 5 se muestra el resultado de algunas de las preguntas de la encuesta de valoración teniendo en cuenta que la escala de puntuación va del 1 (Totalmente en desacuerdo) al 5 (Totalmente de acuerdo).

\section{RESULTADOS DE LA ECUESTA DE VALORACIÓN}

Te ha ayudado a comprender mejor los conceptos de la asignatura involucrados

Se ha visto favorecido el trabajo en grupo

A supuesto mucho tiempo por tu parte

Te ha sido gratificante este modo de trabajo

Ha contribuido a aumentar el grado de satisfacción con la asignatura

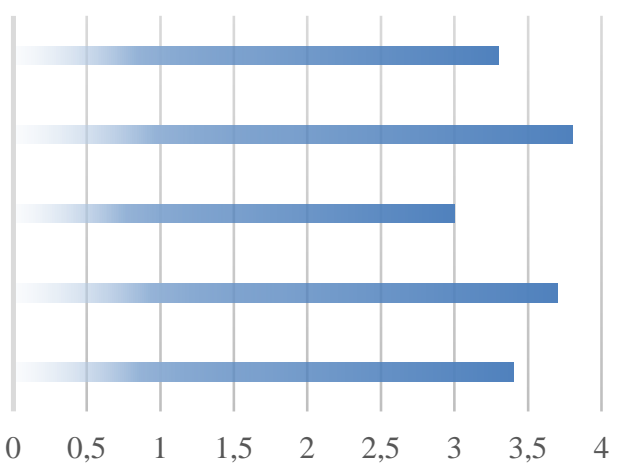

Fig. 5 Resultados de alguna de las preguntas de la encuesta de valoración.

\section{Conclusiones}

Después de un año y medio de proyecto, podemos hacer un breve análisis. En primer lugar, podemos decir que es una experiencia muy positiva y motivadora tanto para estudiantes como para docentes; principalmente, porque ha permitido generar herramientas útiles para involucrar activamente a los estudiantes y acercarles a la asignatura desde la experimentación en el aula. Hemos definido una ficha estándar y sus secciones para completar para cada una de las demostraciones experimentales o proyectos desarrollados por cada profesor. También hemos decidido generar tres prototipos de cada demostración experimental, uno para cada campus. De esta manera, el presente proyecto se estructura como un proyecto de enseñanza Intercampus. Hasta la fecha, todos los participantes en el proyecto ya tienen su primer prototipo desarrollado y los correspondientes tres prototipos. Dicho de otro modo hemos visto que es posible multiplicar los recursos de enseñanza con la creación del catálogo o banco de experimentos mediante un esfuerzo colaborativo entre profesores. Todo este esfuerzo se realiza a coste cero con el objetivo de motivar a los estudiantes y mejorar su aprendizaje. Asimismo, los profesores han encontrado una forma de trabajar a distancia utilizando Google Drive para facilitar el trabajo colaborativo entre diferentes campus. Esto nos está permitiendo generar una cantidad de recursos educativos con un esfuerzo razonable. 
En relación a la implementación de prototipos por parte los estudiantes del Grado en Ingeniería en Diseño Industrial y Desarrollo de Productos, hemos observado y comprobado a partir de los resultados de la encuesta de valoración que la experiencia les resulta gratificante y que les ayuda a asimilar los conceptos de la asignatura de un modo más ameno. También les hace ver que la asignatura de Física puede ser más útil en el diseño de productos, de lo que en general la consideran, lo cual redunda en su mayor motivación a la hora de estudiarla y aprenderla, ya que pasan a considerarla útil.

Por otro lado, la experiencia de mezclar la teoría y la práctica de la asignatura de electricidad en el aula ha sido muy positiva. En particular, la explicación del uso del multímetro en el aula permitió ganar tiempo en las sesiones de laboratorio, que son más cortas y escasas. Los estudiantes estuvieron muy activos y tuvieron con una actitud muy positiva durante la lección. Los comentarios de los estudiantes destacan que el método es agradable y les gusta a asimilar la asignatura.

Como aspecto negativo de estas dos últimas experiencias, hemos observado que la clase puede llegar a volver muy ruidosa; y que por lo tanto, es fundamental tenerla muy bien planificada.

\section{Referencias}

ALBA, J., TORREGROSA, C. y DEL REY, R. (2015). “Aprendizaje basado en proyectos: Primera experiencia en la asignatura de Física del Grado en Ingeniería de Telecomunicación, Sonido e Imagen”. En IN-RED 2015. Valencia: Universitat Politècnica de València.

CALVO, I., LOPEZ-GUEDE, J.M. y ZULUETA, E. (2010). “Aplicando la metodología del maprendizaje basado en Proyectos en la docencia de Ingeniería Técnica en Informática de Gestión”, Revista de Formación e Innovación Educativa Universitaria. vol. 3, № 4, p.166-18.

CURY, A. (2007). Padres Brillantes, Maestros Fascinantes. Ed. Planeta.

FEISEL, L.D. y ROSA, A. J. (2005) "The role of the laboratory in undergraduate engineering education”. J Eng Educ. vol. 94, issue 1, p. 121-130.

FERRER, C. (2012) Demostraciones experimentales de Física para el aula. $<$ http://fisicademos.blogs.uv.es/?page_id=29> [Consulta: 26 de marzo de 2018].

FREIER, G. D. y ANDERSON, F. J. (1996). A demonstration Handbook for Physics $3^{\text {rd }}$ Ed. American Association of Physics Teachers. College Park MD.

GONZÁLEZ, J. y WAGENAAR, R. (2003). “Tuning Educational Structures in Europe. Informe Final” - Proyecto Piloto, Fase 1, Bilbao, Universidad de Deusto.

LLOVERA-SEGOVIA, P., SIMÓN, J. y FUSTER, V. (2015). "Uso de las demostraciones prácticas en las clases de teoría para mejorar el aprendizaje de la Electrotecnia” en 23 Congreso Universitario de Innovación Educativa en las Enseñanzas Técnicas. Valencia. Disponible en $<$ https://innovacioneducativa.upm.es/node/2910> [Consulta: 26 de Marzo del 2018]

VILAPLANA-CERDÁ, R. I., DEL REY-TORMO, R., MANJÓN-HERRERA, F. J., GOMISHILARIO, O., ALBA-FERNÁNDEZ, J., CUENCA-GOTOR, V. P y MONSORIU, J. A. (2017). "Metodologías activas en asignaturas básicas. Creación de un catálogo de demostraciones experimentales o proyectos como recursos didácticos para la motivación de título”. Innovación en la educación superior. PIMES en la ETSID 2016/2017. Valencia: Escuela Técnica Superior de Ingeniería del Diseño. Universidad Politécnica de Valencia. p. 81-86.

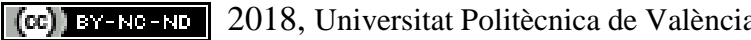

